\title{
MicroRNA-203 As a Stemness Inhibitor of Glioblastoma Stem Cells
}

\author{
Yifan Deng ${ }^{1,2,3}$, Gang Zhu ${ }^{1,3, *}$, Honghai Luo ${ }^{1}$, and Shiguang Zhao ${ }^{2}$
}

\begin{abstract}
Glioblastoma stem cells (GBM-SCs) are believed to be a subpopulation within all glioblastoma (GBM) cells that are in large part responsible for tumor growth and the high grade of therapeutic resistance that is so characteristic of GBM. MicroRNAs (miR) have been implicated in regulating the expression of oncogenes and tumor suppressor genes in cancer stem cells, including GBM-SCs, and they are a potential target for cancer therapy. In the current study, miR-203 expression was reduced in $\mathrm{CD}_{133^{+}}$GBM-SCs derived from six human GBM biopsies. MicroRNA-203 transfected GBM-SCs had reduced capacity for selfrenewal in the cell sphere assay and increased expression of glial and neuronal differentiation markers. In addition, a reduced proliferation rate and an increased rate of apoptosis were observed. Therefore, miR-203 has the potential to reduce features of stemness, specifically in GBM-SCs, and is a logical target for GBM gene therapy.
\end{abstract}

\section{INTRODUCTION}

Glioblastoma (GBM), the most common type of malignant brain tumor, is characterized by highly infiltrative growth and increased resistance to all therapeutic modalities, including surgical resection, radiation, and chemotherapy. Relapse is the norm after comprehensive treatment and prognosis is poor (Omuro and DeAngelis, 2013). Considerable support for the cancer stem cell hypothesis has come from findings in glioblastoma and it is believed that glioblastoma stem cells (GBM-SCs) account for many features of GBM, including its resistance to therapy (Bao et al., 2006; Heywood et al., 2012; Singh et al., 2003).

MicroRNAs (miRNAs) play an important role in regulating gene expression and have a significant association with tumor

\footnotetext{
${ }^{1}$ Department of Neurosurgery, Huizhou Municipal Central Hospital, Huizhou City, Guangdong Province, 516000, The People's Republic of China, ${ }^{2}$ Department of Neurosurgery, The First Affiliated Hospital of Harbin Medical University, Harbin, Heilongjiang Province, The People's Republic of China, ${ }^{3}$ These authors contributed equally to this work.

*Correspondence: dongbasilinger@hotmail.com
}

Received 3 May, 2016; revised 1 July, 2016; accepted 13 July, 2016; published online 3 August, 2016

Keywords: differentiation, glioblastoma stem cells, miR-203, self-renewal ability development (Hayes et al., 2014). They can modify the expression of oncogenes and tumor suppression genes, including within the cancer stem cells, and thereby have a relevant impact on tumor growth and resistance to therapy (Schraivogel et al., 2011; Yi et al., 2008; Zhao et al., 2014).

MicroRNA-203 (miR-203) was previously shown to be expressed at low levels in human glioma tissue (including glioblastoma) compared to normal brain tissue and to also suppress the migration and invasiveness of glioma cells (Dontula et al., 2013). Studies have shown that miR-203 acts as a stemness-inhibiting miRNA, repressing the expression of stem cell factor p63, thereby regulating the properties of skin stem cells (Lena et al., 2008). However, the relationship between miR-203 and GBM-SCs has not been established.

This study is the first to report miR-203 expression specifically in $\mathrm{CD}_{133^{+}}$GBM-SCs and to validate its impact on tumor stem cell characteristics. Notably, we assessed the impact of miR-203 on the ability of CD $133^{+}$GBM-SCs for self-renewal and differentiation of GBM-SC. In addition, we evaluated the effect of miR-203 on overall proliferation, apoptosis, and viability. These results support miR-203 as a valid target in GBM-SCs and gene therapy for glioblastoma.

\section{MATERIALS AND METHODS}

\section{GBM stem cell culture}

Intraoperative GBM tissue samples were obtained from 6 patients (Table 1) at the Department of Neurosurgery, Huizhou Municipal Central Hospital. All tumors were primary lesions. All patients provided written informed consent before participating in this study. The experimental protocol for this study was approved by the Ethics Committee of Huizhou Municipal Central Hospital. GBM tissue from each individual was minced, trypsinized, and resuspended in single cell suspension. After filtra tion and centrifugation, Neurobasal medium supplemented with B27 (diluted to 1:50), $20 \mu \mathrm{g} / \mathrm{L}$ fibroblast growth factor (bFGF), $20 \mu \mathrm{g} / \mathrm{l}$ epidermal growth factor (EGF), and penicillin and streptomycin solution (1:100) was used for cell resuspension. GBMSCs were incubated at $37^{\circ} \mathrm{C}$ with $5 \% \mathrm{CO}_{2}$ in an incubator. All cell culture reagents were purchased from Invitrogen (Thermo Fisher Scientific Co., USA).

\section{Isolation of CD $133^{+}$GBM-SC and purity determination}

When suspended cell spheres reached a diameter of 150-200 $\mu \mathrm{m}$, as observed under a light microscope, they were collected for magnet activated cell sorting (MACS) using CD133 MicroBead Kit for Human Tumor Tissue (Miltenyi Biotec GBMH, 
MicroRNA-203 As a Stemness Inhibitor of Glioblastoma Stem Cells Yifan Deng et al.

Table 1. Demographic characteristics of the patients

\begin{tabular}{ccclc}
\hline Patient \# & Age (yr) & Sex & Tumor location & $\begin{array}{c}\text { Therapy before } \\
\text { resection }\end{array}$ \\
\hline 1 & 59 & F & Right temporal lobe & \\
2 & 24 & M & Left frontal lobe & Anti-epileptics \\
3 & 52 & F & Right temporal lobe & \\
4 & 30 & M & Right frontal lobe & Steroids \\
5 & 36 & F & Right frontal lobe & Anti-epileptics \\
6 & 45 & M & Left temporal lobe & \\
\hline
\end{tabular}

Germany) to select for $\mathrm{CD} 133^{+}$cells according to the manufacturer's instruction and based on the protocol of Beier et al. (Beier et al., 2007; 2008). Sorted CD133 ${ }^{+}$cells were counted, spun at $1,000 \mathrm{rpm}$ for $5 \mathrm{~min}\left(37^{\circ} \mathrm{C}\right)$ and the cell pellet collected. Pre-cooled $\left(4^{\circ} \mathrm{C}\right)$ cell sorting solution was added to resuspend the cells $\left(200 \mu\right.$ l per $10^{8}$ cells). Cells were incubated for 5 min with $\mathrm{Fc}$ receptor (FcR) blocking reagent $\left(100 \mu \mathrm{l}\right.$ per $10^{8}$ cells) to inhibit non-specific binding between anti-CD133 antibody and CD133 cells. Anti-CD133 MicroBeads (100 $\mu$ l per $10^{8}$ cells) were added and incubated with the cells at $4^{\circ} \mathrm{C}$ for $30 \mathrm{~min}$. Cell sorting solution was added at a final concentration of $10^{8}$ cells $/ \mathrm{ml}$ to wash the cells, followed by centrifugation at 1,000 rpm for $5 \mathrm{~min}$ and resuspension of the pellet in cell sorting solution $\left(500 \mu \mathrm{l}\right.$ per $10^{8}$ cells). The cell sorting column was placed in the magnetic field of a suitable MACS separator and rinsed with $500 \mu \mathrm{l}$ cell sorting solution to pre-wet the separation column by gravity flow. The cell suspension with anti-CD133 MicroBeads was applied to the cell sorting column and allowed to flow through, followed by two additional rinses with cell sorting. Flow-through containing unlabeled cells (i.e., CD133- cells) was collected. Cell sorting column was then removed from the MACS separator and placed in a $15 \mathrm{ml}$ centrifugation tube, followed by an additional $2 \mathrm{ml}$ of cell sorting solution for elution of $\mathrm{CD} 133^{+}$cells and centrifugation at $1,000 \mathrm{rpm}$ for $5 \mathrm{~min}$ at $37^{\circ} \mathrm{C}$. Eluted CD133+ cells were further incubated in stem cell culture medium. Purity of $\mathrm{CD} 133^{+}$and $\mathrm{CD} 133^{-}$cells was determined by flow cytometry using $50 \mu \mathrm{l}$ of human monoclonal CD133/2 (293C3)-PE antibody (Miltenyi Biotec GBMH) or $50 \mu$ l of isotype control mlgG2b-PE antibody (Caltag Laboratories, USA) and the FACSCalibur flow cytometer (BD Bioscience, USA).

\section{Immunofluorescence of GBM-SCs}

To verify the GBM-SC status, immunofluorescence (IF) staining with the stem cell markers, CD133, and nestin was performed after sorting the GBM-SCs. After differentiation into astrocytic and neuronal lineages with DMEM high glucose medium containing $10 \%$ fetal bovine serum (Invitrogen), cells were stained with the astrocyte markers, glial fibrillary acidic protein (GFAP), and neuronal microtubule-associated protein 2 (MAP2) using the following steps: GBM-SCs were fixed with $4 \%$ paraformaldehyde, followed by washing and permeabilization in $0.4 \%$ Triton X-100. After sequential incubations with individual primary antibodies and the corresponding secondary antibodies, GBM-SCs were counter-stained with 4',6-diamidino2-phenylindole (DAPI) solution to visualize the nuclei. GBMSCs from different groups were mounted on glass slides and analyzed for cytomorphological protein expression by fluorescence microscopy after image capture. Individual antibodies used for IF staining were: rabbit anti-human CD133 monoclonal antibody (1:200, Abcam, UK); mouse anti-human nestin monoclonal antibody (1:200, Merck Millipore, Germany); rabbit anti- human GFAP monoclonal antibody (1:5000, Abcam); mouse anti-human MAP2 monoclonal antibody (1:500, Abcam); Alexa fluor 488 goat anti-rabbit IgG (1:200, Molecular Probes, USA); and Alexa fluor 594 goat anti-mouse IgG (1:300, Molecular Probes).

Real time-polymerase chain reaction (RT-PCR) to detect miR-203 expression in CD133 ${ }^{+}$GBM-SC sorted cells

Expression of miR-203 was detected using a fluorescent TaqMan probe following the manufacturer's protocol in the TaqMan microRNA assay kit (Applied Biosystems Inc., Life Technologies Co., USA). U6 snRNA was used as an internal reference gene.

\section{Cell transfection}

Human mature miR-203 analog and a random sequence used as the "normal control" (NC) were purchased from Shanghai GenePharma Co., Ltd. (China), with the miR-203 sequence of 5'-GAUCACCAGGAUUUGUAAAGUG-3' and the NC sequence of $5^{\prime}$-UUCUCCGAACGUGUCACGUTT-3'. Cell transfection was performed using the Lipofectamine ${ }^{\circledR}$ RNAiMAXTM transfection reagent (Invitrogen) according to the manufacturer's protocol. BLOCK-iT ${ }^{\mathrm{TM}}$ Alexa Fluor ${ }^{\circledR}$ red fluorescent oligo (Invitrogen) was used as the transfection tracer to determine transfection efficiency. The transfected GBM-SCs with increased levels of miR-203 were identified using TaqMan-based qRT-PCR

Sphere-forming assays to detect the effect of miR-203 on self-renewal capacity of GBM-SCs

The sphere-forming assay was performed as previously described (Zhao et al., 2013). Briefly, 1,000 GBM-SCs, each in the miR-203 transfected and NC groups, were individually seeded in 96-well plates. The number of GBM-SCs that formed spheres was determined after 7 days. This was the first phase. Subsequently, GBM-SCs of the two groups were harvested, transfected, and cultured again for 7 days (at the same cell density). The number of GBM-SCs that formed spheres in this second phase was determined. The percentage of sphereforming cells in the miR-203 transfected and the NC groups was calculated.

RT-PCR detection of mRNA expression of CD133, nestin, GFAP, and MAP2 in two groups of GBM-SCs after

transfection

RT-PCR was used to detect the mRNA expression of $C D 133$, nestin, GFAP, and MAP2 in the two groups of GBM-SCs 72 hours after transfection. Total RNA from GBM-SCs in each group was isolated using the acid guanidinum thiocyanatephenol-chloroform extraction method as previously described (Chomczynski and Sacchi, 2006). Total RNA (1000 ng) was used as a temperate for RT-PCR using SYBR Green PCR Master Mix reagent kit (Applied Biosystems Inc., Life Technologies Co.). Beta-actin ( $\beta$-actin) was used as the internal reference in the reaction. The $2^{-\Delta \Delta C T}$ method was used to calculate the relative mRNA expression levels. PCR primer sequences used in this study follow: CD133 (forward primer: CAGGTAAGAACCCGGATCAA, reverse primer: TCAGATCTG TGAACGCCTTG); nestin (forward primer: GCAGCAGGAAATA TGGGAAG, reverse primer: TCTCATGGCTCTGGTTTTCC), GFAP (forward primer: ACACCAAGTCTGTGTCAGAAG, reverse primer: CTCCTTATTGACCTCTCCATC), MAP2 (forward primer: GCAGTTCTCAAAGGCTAGAC, reverse primer: TGATCGTGG AACTCCATCT), and $\beta$-actin (forward primer: TGCGTGACATT 
A

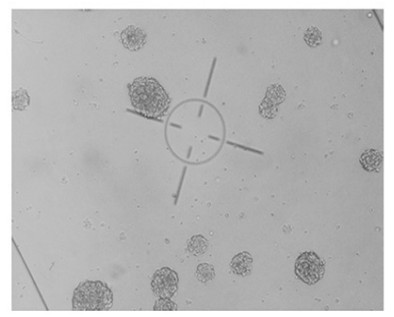

B

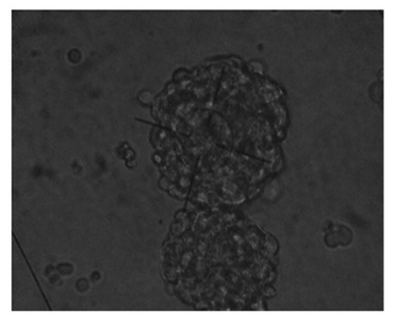

C

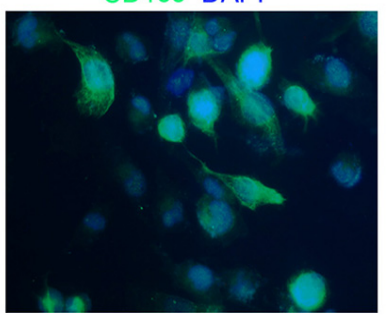

D

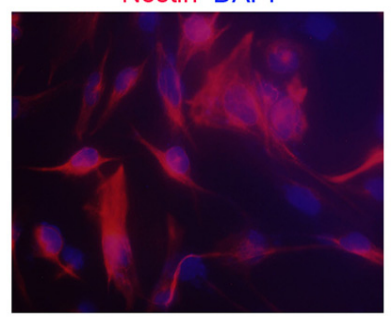

E

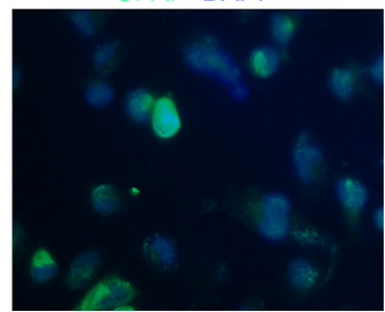

$\mathbf{F}$

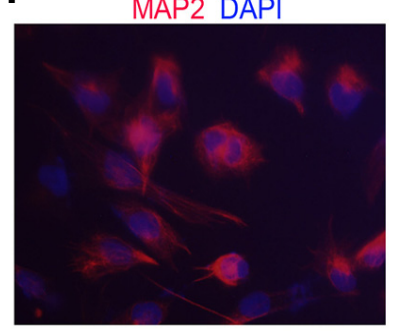

Fig. 1. Identification and morphological features of glioblastoma stem cells (GBM-SCs). (A) GBM cell sphere formation (10× magnification); (B) GBM cell sphere formation (20x magnification); (C, D) Representative images of GBM-SCs showing the widely expressed CD133 and Nestin in GBM-SCs (40x magnification); and $(E, F)$ Representative images of GBM-SCs showing the widely expressed glial fibrillary acidic protein (GFAP) and microtubuleassociated protein 2 (MAP2) in GBM-SCs (40× magnification).
AAGGAGAAG, reverse primer: GCTCGTAGC TCTTCTCCA). Reaction conditions of reverse transcription of RNA to CDNA were as follows: annealing at $27^{\circ} \mathrm{C}$ for $9 \mathrm{~min}$; extension at $37^{\circ} \mathrm{C}$ for $120 \mathrm{~min}$; and termination at $85^{\circ} \mathrm{C}$ for 5 min followed by cooling at $4^{\circ} \mathrm{C}$. All reverse transcribed products were stored at $-20^{\circ} \mathrm{C}$ for later use. Amplification conditions of fluorescence-based quantitative PCR are as follows: initial denaturation at $93^{\circ} \mathrm{C}$ for 4 min followed by 40 cycles of denaturation at $93^{\circ} \mathrm{C}$ for $20 \mathrm{~s}$, annealing at $60^{\circ} \mathrm{C}$ for $30 \mathrm{~s}$, and extension at $70^{\circ} \mathrm{C}$ for $30 \mathrm{~s}$.

Cell counting Kit-8 (CCK-8) assay to evaluate the effect of miR-203 on GBM-SCs proliferation

Following transfection (at 24, 48, 72, 96, and $120 \mathrm{~h}$ ), GBM-SCs from both of the groups were cultured with the CCK-8 solution for $4 \mathrm{~h}$ (Dojindo Molecular Technologies, Inc., Japan) as described in the manufacturer's protocol. Absorbance values were measured for each group of GBM-SCs using the Biotek microplate reader (absorbance at a wavelength of $450 \mathrm{~nm}$ ). Cell viability was expressed as the percent of control and calculated with the following formula Viability $=\mathrm{AE} / \mathrm{AC} \times 100 \%$ (where $\mathrm{AC}$ $=$ absorbance value of the normal control group, and $A E=a b-$ sorbance value of the experimental group).

\section{Flow cytometry to detect apoptosis of GBM-SCs}

GBM-SCs in the two groups were stained with annexin $V$ conjugated to green-fluorescent FITC dye 3 days after transfection. Apoptosis of GBM-SCs in different groups was detected by FACSCalibur flow cytometer (BD Bioscience, USA), and the data was analyzed using Cell Quest 3.3 software.

\section{Statistical analysis}

SPSS 13.0 software (SPSS Inc., USA) was used for statistical analysis. Data are presented as mean \pm standard deviation. ANOVA was used for comparisons between multiple groups. Fisher's least significant difference test (LSD-t) was used for pairwise comparisons. T test was used for comparison between two groups. $P<0.05$ was considered statistically significant.

\section{RESULTS}

Culture and identification of GBM-SCs

After incubating primary cells in neural stem cell culture medium for 3-4 days, a large number of suspended cell spheres were observed. CD133 ${ }^{+}$cells isolated by MACS were considered as the GBM-SCs in this study. They were cultured in stem cell medium to form stem cell-like neurospheres. After 7-8 days of culturing, round or oval-shaped cell spheres appeared with a diameter of approximately 100-200 $\mu \mathrm{m}$ with sharp cell edges and good-quality refraction (Figs. $1 \mathrm{~A}$ and $1 \mathrm{~B}$ ).

IF staining showed that GBM-SCs expressed two stem cell protein markers, CD133 and nestin (Figs. 1C and 1D), indicating that the cells were bona fide GBM-SCs from GBM tissue. To induce differentiation, cells were grown in DMEM (Invitrogen, Thermo Fisher Scientific Co., USA) supplemented with $10 \%$ FBS (Invitrogen) for 5 days. Cell morphology changed when cultured in this differentiation medium, and dendrites were observed. Cells cultured in differentiation medium also expressed GFAP and MAP2, corroborating the multilineage differentiation and stem-cell like nature of the isolated cell population (Figs. 1E and $1 \mathrm{~F}$ ).

\section{miR-203 expression in GBM-SCs}

After cell sorting for $\mathrm{CD}_{133^{+}}$cells by MACS, purity was assessed as more than $97 \%$ (Fig. 2A). Quantitative real-time PCR (RT-PCR) results indicated that miR-203 was expressed at higher levels in CD133- cells than in $\mathrm{CD}^{-} 133^{+}$cells and the difference (expressed as "fold" increase and normalized to U6 snRNA) was $6.497 \pm 0.778(P=0.003)$ in patient $\# 1 ; 2.843 \pm$ $0.741(P=0.025)$ in patient \#2; $1.983 \pm 0.315(P=0.0163)$ in patient \#3; $6.373 \pm 2.971(P=0.0443)$ in patient \#4; $4.327 \pm$ $1.592(P=0.0343)$ in patient $\# 5$; and $8.213 \pm 2.768(P=$ 0.0229 ) in patient \#6 (Fig. 2B). The average fold increase of all six patients was $5.039 \pm 2.394(P=0.0045)$.

At $24 \mathrm{hr}$ after transfection with BLOCK-іT'М Alexa Fluor ${ }^{\circledR}$ red fluorescent oligo, transfection efficiency was determined to be $85.93 \pm 2.55 \%$ as measured by fluorescence microscopy. Compared with NC-transfected GBM-SCs, miR-203 was expressed 

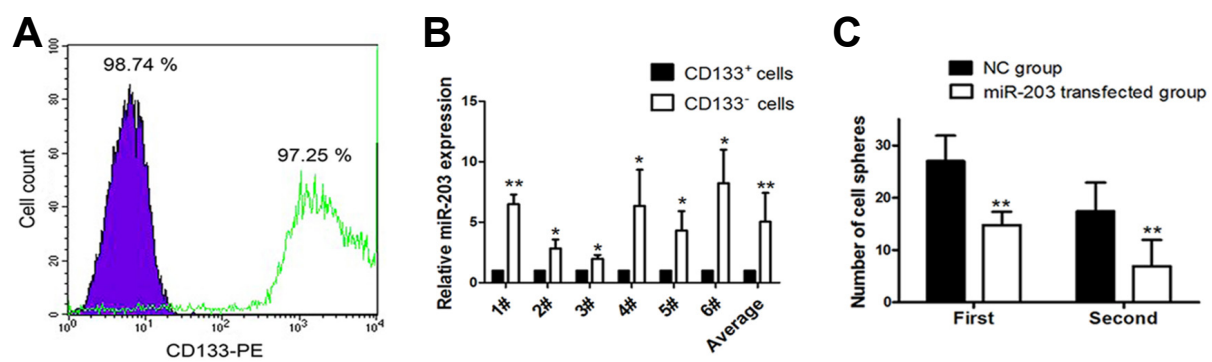

Fig. 2. Relative expression of microRNA-203 (miR-203) in glioblastoma stem cells (GBM$\mathrm{SCs}$ ) sphere assay results. (A) Flow cytometry analysis of the neuronal stem cell marker, CD133-PE, in cultured GBMSCs. The purple peak represents the result of CD133 cells and the white peak with the solid green line represents the result of $\mathrm{CD} 133^{+}$cells. Percentages of $\mathrm{CD}_{133^{+}}$and $\mathrm{CD} 133^{-}$cells are indicated. (B) Relative expression of miR-203 in $\mathrm{CD}_{133^{+}}$and $\mathrm{CD} 133^{-}$cells measured by RT-PCR. \#1 to \#6 represents GBM-SCs obtained from individual GBM tissues from 6 patients. (C) Changes in self-renewal capacity of GBM-SCs after miR-203 transfection measured by the sphere assay. Y-axis represents the number of cell spheres formed and X-axis represents the order of GBM sphere formation; NC represents the normal control GBM-SC group and miR-203 represents the miR-203-transfected GBM-SC group. In (B) and (C), double asterisks "**" represent $P<0.01$ between two groups, and single asterisk "*" represents $P<0.05$ between two groups.

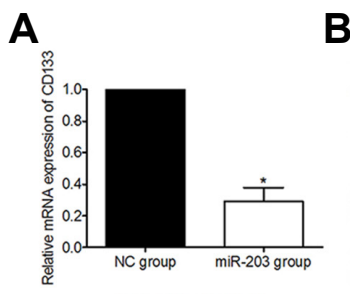

E
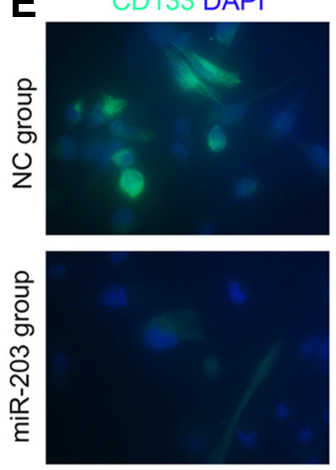

B
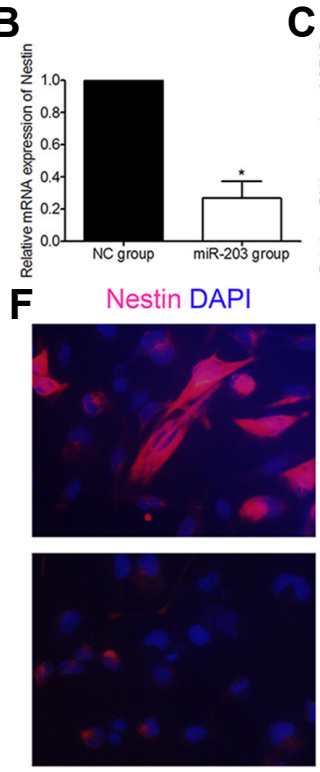

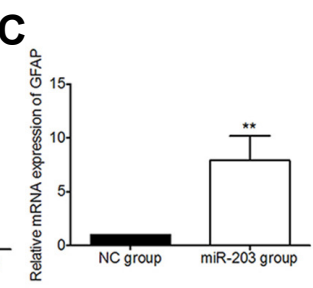

G
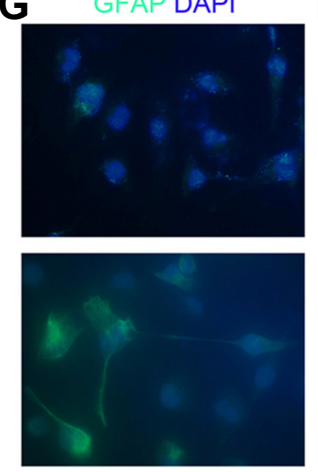

$\mathbf{D}$

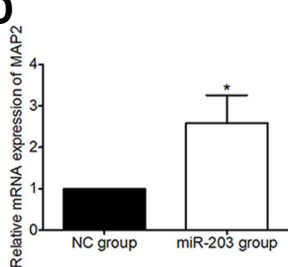

$\mathbf{H}$
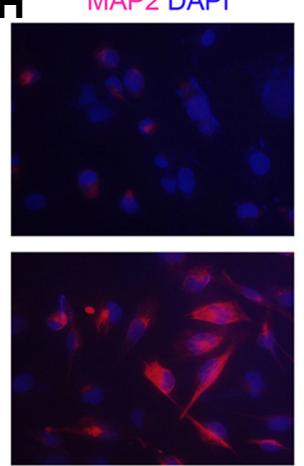

Fig. 3. mRNA and protein expression in CD133, nestin, expressed glial fibrillary acidic protein (GFAP), and microtubule-associated protein 2 (MAP2) in glioblastoma stem cells (GBMSCs) after microRNA-203 (miR-203) transfection. (A-D) Relative mRNA expression. (E-H) Representative immunofluorescence images (40x magnification). Note: $\mathrm{NC}$ represents the normal control GBM-SC group and miR-203 represents the miR-203 transfected GBMSC group; double asterisks “**” represent $P<0.01$ between two groups, and asterisk "*" represents $P<$ 0.05 between two groups. at higher levels in the miR-203 transfected cells after transfection and the difference (expressed as "fold" increase and normalized to U6 snRNA) was $102.33 \pm 11.50(P=0.002)$ after 24 $\mathrm{h}, 53.67 \pm 5.69(P=0.002)$ after $48 \mathrm{~h}$, and $5.33 \pm 2.52(P=$ 0.048 ) after $72 \mathrm{~h}$.

\section{Effect of miR-203 overexpression on the self-renewal capacity of GBM-SCs}

Seven-days after transfection with miR-203, fewer cell spheres cells $(27.0 \pm 4.8, P=0.0090$, Fig. $2 \mathrm{C})$ and they tended to be smaller in size. When compared with NC-transfected CD133 cells (set at $100 \%$ ), only $67.84 \pm 13.79 \%$ of the miR203transfected $\mathrm{CD} 133^{+}$cells formed spheres in the first phase of the assay. In the second phase of the cell sphere formation assay, miR203-transfected $\mathrm{CD} 133^{+}$cells again formed fewer cell spheres $(6.9 \pm 5.0)$ than NC-transfected CD133 ${ }^{+}$cells $(17.4$ $\pm 5.5, P=0.0027$, Fig. $2 \mathrm{C}$ ). The relative number of cells forming spheres in this phase in the miR-203 transfected group was $30.53 \pm 8.91 \%$ of the NC group. These findings suggest that
miR-203 significantly inhibits the self-renewal capacity of GBMSCs.

Effect of miR-203 on the expression of multilineage marker expression of GBM-SCs

At 3 days following transfection, RT-PCR was used to detect CD133, nestin, GFAP, and MAP2 mRNA in the two groups of GBM-SCs. mRNA expression of CD133 and nestin was significantly lower in the miR-203 transfected vs. the NC group while mRNA expression of GFAP and MAP2 were significantly higher in the miR-203 transfected vs. the NC group (Figs. 3A-3D). Results of mRNA expression CD133, nestin, GFAP, and MAP2 (Figs. 3A-3D) were consistent with the IF staining (Figs. 3E-3H).

Effect of miR-203 on cell proliferation and apoptosis of GBM-SCs

Compared with NC-transfected GBM-SCs, absorbance in the CCK-8 assay was lower in the miR-203 transfected cells at 24 h, $48 \mathrm{~h}, 72 \mathrm{~h}, 96 \mathrm{~h}$, and $120 \mathrm{~h}$ following transfection $(P=0.04 ; P$ 
A

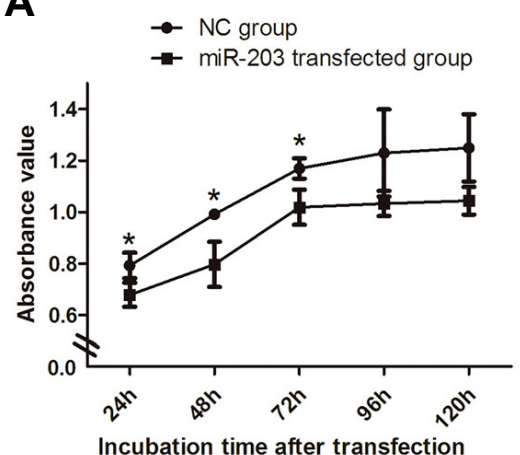

B

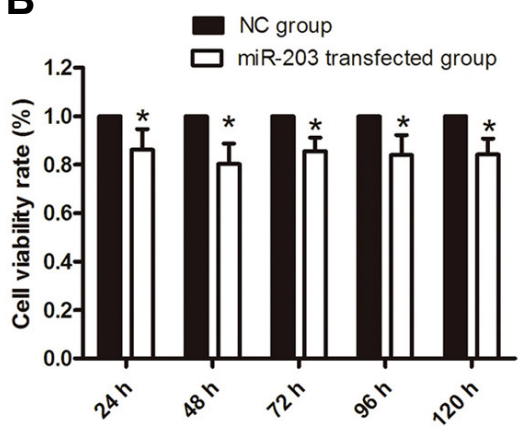

C

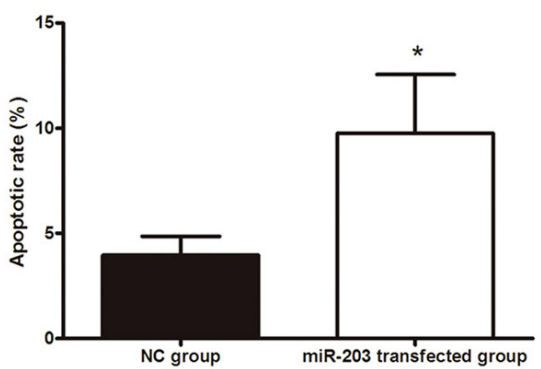

Fig. 4. Effect of miR-203 on the growth and apoptosis of GBM-SCs. (A) Histogram showing absorbance values of GBM-SCs in NC and miR203 transfected groups at different time points of incubation after transfection. (B) Histogram showing the cell viability of GBM-SCs (expressed as \% of NC) in the miR-203 transfected group (right panel) at different time points of incubation after transfection. (C) Histogram showing the relative amount of apoptotic GBM-SCs (expressed as \% of total cells) in NC and miR-203 transfected groups. Asterisk “*” represents $P<0.05$ between the two groups.

$=0.008 ; P=0.04 ; P=0.13$; and $P=0.07$, respectively) (Fig. $4 \mathrm{~A})$. Accordingly, cell viability (expressed as percent of control) was also lower in miR-203 transfected GBM-SCs when compared with NC-transfected GBM-SCs $(P=0.05 ; P=0.02 ; P=$ $0.012 ; P=0.03$; and $P=0.01$, respectively) (Fig. 4B). Flow cytometry was performed to test if increased cell death contributed to reduced cell viability. The results demonstrated that the apoptotic rate of GBM-SCs in the miR-203 transfected group was significantly higher than in the NC-transfected group $(9.74$ $\pm 2.81 \%$ vs. $3.95 \pm 0.91 \%, P=0.027$, Fig. 4 C).

\section{DISCUSSION}

An increasing body of research supports an important role for tumor stem cells in tumorigenesis, tumor growth, metastases, tumor recurrence, and tumor resistance to therapy (Clevers, 2011; Dean et al., 2005; Visvader and Lindeman, 2008). Consequently, targeting tumor stem cells is seen as an attractive therapeutic strategy in oncology. Despite the fact that CD133 does detect all GBM-SC populations, indicated by the existence of CD133- GBM-SCs, CD133 remains a useful marker for GBM-SCs (Bao et al., 2006; Singh et al., 2003). However, in GBM tissue, CD133+ cells have consistently been shown to possess stem cell properties and $\mathrm{CD}_{133^{+}}$gliomas are associated with higher proliferation rates than gliomas lacking $\mathrm{CD}_{133^{+}}$ GBM-SCs (Beier et al., 2007). Therefore, the current study focused solely on CD133 ${ }^{+}$GBM-SCs.

miRNAs have been broadly implied in regulating the expression of oncogenes and tumor suppressor genes in tumor stem cells, including GBM-SCs (Schraivogel et al., 2011; Zhao et al., 2014). A previous study demonstrated that the expression of miR-203 in glioma cells was low, and that transfection of miR203 suppressed cell proliferation, migration, and invasiveness via modulation of Robo1 (roundabout, axon guidance receptor, homolog 1)/ERK (extracellular signal-regulated kinases)/MMP9 (metalloproteinase-9) signaling (Dontula et al., 2013). However, the study did not address if miR-203 expression is reduced specifically in GBM-SCs, arguably the most relevant cell subpopulation in GBM for therapeutic purposes. The current study isolated $\mathrm{CD} 133^{+}$from GBM biopsies to obtain a relative-
Iyhomogeneous population of GBM-SCs. In addition to CD133 immunostaining, cell spheres also stained positive for nestin and upon differentiation, and individual cells within the spheres expressed GFAP or MAP2, which confirmed the stemness and multilineage differentiation of the isolated $\mathrm{CD}_{133^{+}}$cell population.

When compared with the CD133- cells from the same tumors $\mathrm{CD}_{133^{+}}$cells had reduced expression of miR-203, confirming that the reduced expression of miR-203 in GBM is specific for the GBM-SC population. Moreover, overexpression of miR-203 in the $\mathrm{CD}_{133^{+}}$led to a significant reduction of self-renewal in the cell sphere assay and to increased apoptosis. Consequently, viability was reduced after miR-203 transfection, which suggests that miR-203 impairs the ability of GBM-SCs for selfrenewal and proliferation. In addition, at the mRNA level, expression of the stem cell makers, CD133, and nestin was reduced after miR-203 transfection, whereas expression of GFAP and MAP2 was increased. These results suggest that the effect of miR-203 on GBM-SCs is, at least in part, driven by forcing GBM-SCs into differentiation. The exact mechanism through which miR-203 exerts its effects in GBM-SCs remains to be determined. A previous study by Chen et al. reported that miR203 directly downregulated phospholipase D2 (PLD2) protein, thereby inhibiting proliferation of a GBM cell line and reducing cell viability by $60 \%$ (Chen et al., 2014). Thus, PLD2 is a potential target for miR-203 in GBM-SCs. It is noteworthy that in the current study the effect of miR-203 on viability (an approximate $20 \%$ reduction) and apoptosis (increase from approximately $4 \%$ to approximately $10 \%$ ) was more modest than in the U251 cell line tested by Chen et al. (Chen et al., 2014). However, the effect of miR-203 in the cell sphere assay was pronounced (an approximate $70 \%$ reduction of sphere formation in the second round). This seeming discrepancy could be explained by miR203 predominantly reducing the capacity for self-renewal rather than proliferation, per se. This interpretation is in line with the observation of increased expression of GFAP and MAP2 after miR-203 transfection, which indicates a tendency towards increased differentiation. One limitation of the current study is that the effects were tested in an ex vivo setting and it is not clear if the susceptibility of GBM-SCs to miR-203 expression is affected by the culture condition. Hence, the effect of miR-203 on 
GBM-SCs in vivo needs to be explored in future studies. Another consideration is that any future therapy aimed at increasing miR-203 expression in GBM-SCs may only be effective in GBMs with $\mathrm{CD}_{133^{+}}$GBM-SCs and low expression of miR-203.

\section{ACKNOWLEDGMENTS}

We would like to thank LetPub (www.letpub.com) for its linguistic assistance during the preparation of this manuscript. This study was supported by the Medical Scientific Research Foundation of Guangdong Province, China (No. B2014417).

\section{REFERENCES}

Bao, S., Wu, Q., McLendon, R.E., Hao, Y., Shi, Q., Hjelmeland, A.B., Dewhirst, M.W., Bigner, D.D., and Rich, J.N. (2006). Glioma stem cells promote radioresistance by preferential activation of the DNA damage response. Nature 444, 756-760.

Beier, D., Hau, P., Proescholdt, M., Lohmeier, A., Wischhusen, J., Oefner, P.J., Aigner, L., Brawanski, A., Bogdahn, U., and Beier, C.P. (2007). CD133(+) and CD133(-) glioblastoma-derived cancer stem cells show differential growth characteristics and molecular profiles. Cancer Res. 67, 4010-4015.

Beier, D., Wischhusen, J., Dietmaier, W., Hau, P., Proescholdt, M., Brawanski, A., Bogdahn, U., and Beier, C.P. (2008). CD133 expression and cancer stem cells predict prognosis in high-grade oligodendroglial tumors. Brain Pathol. 18, 370-377.

Chen, Z., Li, D., Cheng, Q., Ma, Z., Jiang, B., Peng, R., Chen, R., Cao, Y., and Wan, X. (2014). MicroRNA-203 inhibits the proliferation and invasion of U251 glioblastoma cells by directly targeting PLD2. Mol. Med. Rep. 9, 503-508.

Chomczynski, P., and Sacchi, N. (2006). The single-step method of RNA isolation by acid guanidinium thiocyanate-phenolchloroform extraction: twenty-something years on. Nat. Protoc. 1, 581-585.

Clevers, H. (2011). The cancer stem cell: premises, promises and challenges. Nat. Med. 17, 313-319.
Dean, M., Fojo, T., and Bates, S. (2005). Tumour stem cells and drug resistance. Nat. Rev. Cancer 5, 275-284.

Dontula, R., Dinasarapu, A., Chetty, C., Pannuru, P., Herbert, E., Ozer, H., and Lakka, S.S. (2013). MicroRNA 203 modulates Glioma cell migration via Robo1/ERK/MMP-9 signaling. Genes Cancer 4, 285-296.

Hayes, J., Peruzzi, P.P., and Lawler, S. (2014). MicroRNAs in cancer: biomarkers, functions and therapy. Trends Mol. Med. 20, 460-469.

Heywood, R.M., Marcus, H.J., Ryan, D.J., Piccirillo, S.G., AlMayhani, T.M., and Watts, C. (2012). A review of the role of stem cells in the development and treatment of glioma. Acta Neurochir (Wien) 154, 951-969; discussion 969.

Lena, A.M., Shalom-Feuerstein, R., Rivetti di Val Cervo, P., Aberdam, D., Knight, R.A., Melino, G., and Candi, E. (2008). miR-203 represses 'stemness' by repressing DeltaNp63. Cell Death Differ. $15,1187-1195$.

Omuro, A., and DeAngelis, L.M. (2013). Glioblastoma and other malignant gliomas: a clinical review. JAMA 310, 1842-1850.

Schraivogel, D., Weinmann, L., Beier, D., Tabatabai, G., Eichner, A., Zhu, J.Y., Anton, M., Sixt, M., Weller, M., Beier, C.P., et al. (2011). CAMTA1 is a novel tumour suppressor regulated by miR-9/9* in glioblastoma stem cells. EMBO J. 30, 4309-4322.

Singh, S.K., Clarke, I.D., Terasaki, M., Bonn, V.E., Hawkins, C. Squire, J., and Dirks, P.B. (2003). Identification of a cancer stem cell in human brain tumors. Cancer Res. 63, 5821-5828.

Visvader, J.E., and Lindeman, G.J. (2008). Cancer stem cells in solid tumours: accumulating evidence and unresolved questions. Nat. Rev. Cancer 8, 755-768.

Yi, R., Poy, M.N., Stoffel, M., and Fuchs, E. (2008). A skin microRNA promotes differentiation by repressing 'stemness'. Nature 452 , 225-229.

Zhao, S., Deng, Y., Liu, Y., Chen, X., Yang, G., Mu, Y., Zhang, D., Kang, J., and Wu, Z. (2013). MicroRNA-153 is tumor suppressive in glioblastoma stem cells. Mol. Biol. Rep. 40, 2789-2798.

Zhao, B., Bian, E.B., and Li, J. (2014). New advances of microRNAs in glioma stem cells, with special emphasis on aberrant methylation of microRNAs. J. Cell Physiol. 229, 1141-1147. 